TRANSACTIONS OF THE

AMERICAN MATHEMATICAL SOCIETY

Volume 350, Number 7, July 1998, Pages 2925-2937

S $0002-9947(98) 02276-4$

\title{
A SINGULAR QUASILINEAR ANISOTROPIC ELLIPTIC BOUNDARY VALUE PROBLEM. II
}

\author{
Y. S. CHOI AND P. J. MCKENNA
}

\begin{abstract}
Let $\Omega \subset \mathbf{R}^{N}$ with $N \geq 2$. We consider the equations

$$
\begin{aligned}
\sum_{i=1}^{N} u^{a_{i}} \frac{\partial^{2} u}{\partial x_{i}^{2}}+p(\mathbf{x}) & =0 \\
\left.u\right|_{\partial \Omega} & =0
\end{aligned}
$$

with $a_{1} \geq a_{2} \geq \ldots . \geq a_{N} \geq 0$ and $a_{1}>a_{N}$. We show that if $\Omega$ is a convex bounded region in $\mathbf{R}^{N}$, there exists at least one classical solution to this boundary value problem. If the region is not convex, we show the existence of a weak solution. Partial results for the existence of classical solutions for non-convex domains in $\mathbf{R}^{2}$ are also given.
\end{abstract}

\section{INTRODUCTION}

The purpose of this paper is to answer two of the questions left open in [2]. Recall that that paper began the study of the boundary value problem

$$
\begin{gathered}
u^{a} u_{x x}+u^{b} u_{y y}+p(\mathbf{x})=0, \\
\left.u\right|_{\partial \Omega}=0,
\end{gathered}
$$

in a smooth bounded convex domain $\Omega$ in $\mathbf{R}^{2}$. Here $a$ and $b$ are non-negative, but are not equal, and the function $p$ was positive and continuous on the closure of $\Omega$.

There were two motivations for the study of this equation. First, there was a large and interesting literature in the isotropic case where $a=b$, even for domains in higher dimensions. (See, for example $[5,6,3,9,10]$ for references.) When (1.1)(1.2) arose naturally from discussions involving fluid dynamics [1], it was natural to wonder how much of the existing theory would carry over to the anisotropic case.

The short answer to this question was that not much did. It was proved that a classical solution exists, but $\Omega$ had to be made convex and restricted to $R^{2}$.

Monotone iteration techniques in the usual treatment of semilinear elliptic equations fail in this case. The solution has to be extracted by a compactness argument. The proof in [2] made use of an interior estimate that is valid only in two dimensions. More seriously, the convex domain assumption is crucial in the construction of an upper solution satisfying the boundary condition. This ensures that the constructed solution will satisfy the boundary conditions.

In this paper, we remedy two of the basic flaws in the earlier paper. First, we remove the restriction that the dimension be restricted to two, even though in

Received by the editors August 6, 1996.

1991 Mathematics Subject Classification. Primary 35J25.

Key words and phrases. Harnack inequality, singular, subsolution, supersolution.

(C)1998 American Mathematical Society 
this case, we retain the convexity condition, and show that a classical solution still exists.

Let $\Omega \subset \mathbf{R}^{N}$ with $N \geq 2$. We therefore consider the equations

$$
\begin{gathered}
\sum_{i=1}^{N} u^{a_{i}} \frac{\partial^{2} u}{\partial x_{i}^{2}}+p(\mathbf{x})=0 \\
\left.u\right|_{\partial \Omega}=0
\end{gathered}
$$

with $a_{1} \geq a_{2} \geq \ldots \geq a_{N} \geq 0$ and $a_{1}>a_{N}$. This requires a new interior apriori estimate. Then we have the following direct generalization of the main result in [2].

Theorem 1. Let $\Omega \subset \mathbf{R}^{N}, N \geq 1$, be a bounded convex domain with smooth boundary $\partial \Omega$ (of class $C^{2+\alpha}, 0<\alpha<1$ ) and let $a_{1} \geq a_{2} \geq \ldots \geq a_{N} \geq 0$ with $a_{1}>a_{N}$. If $p \in C^{\alpha}(\bar{\Omega}), M \geq p(\mathbf{x}) \geq m>0$ for all $\mathbf{x} \in \bar{\Omega}$, then there exists a function $u \in C^{2+\alpha}(\Omega) \cap C(\bar{\Omega})$ such that $\psi(\mathbf{x}) \geq u(\mathbf{x})>0$ for all $\mathbf{x} \in \Omega$ and $u$ is a solution of (1.3)-(1.4). Here $\psi$ is the solution to equations (1.5)-(1.6).

The second flaw in the earlier paper is that the existence result is restricted to convex domains. This we improve with a result about non-convex domains. Perhaps what is most interesting about this result is what we don't prove. Given the previous discussion, one might expect that we would prove theorem 1 in the case of non-convex domains. Instead, we prove a weak version of theorem 1, namely

Theorem 2. Let all the assumptions in theorem 1 hold, except for the requirement on the convexity of $\Omega$. Then there exists a function $u \in C^{2+\alpha}(\Omega)$ such that $\psi(\mathbf{x}) \geq$ $u(\mathbf{x})>0$ for all $\mathbf{x} \in \Omega$ and $u$ is a solution of (1.3) with $u^{\theta} \in H_{0}^{1}(\Omega)$, where $\theta=\frac{a_{1}+1}{2}>0$. Here $\psi$ is the solution to equations (1.5)-(1.6).

Our final theorem is a very partial result which tries to reconcile theorems 1 and 2. In this case, we show that if the dimension is restricted to 2 , and severe retrictions are imposed on the degeneracy, it is possible to get full classical solutions in the non-convex case.

Theorem 3. Let all the assumptions in theorem 1 hold, except that $\Omega$ is a nonconvex bounded smooth domain in $\mathbf{R}^{2}$. Also, assume $a \equiv a_{1}+a_{2}<1$. Then there exists a function $u \in C^{2+\alpha}(\Omega) \cap C(\bar{\Omega})$ such that $u(\mathbf{x})>0$ for all $\mathbf{x} \in \Omega$ and $u$ is a solution of (1.3)-(1.4).

In section 2 we will study a regularized problem which approximates equations (1.3)-(1.4). In section 3 we will describe a comparison principle for quasilinear elliptic problems. In section 4, results from the previous two sections are employed to prove theorem 1 , which is valid in convex domains. We deal with non-convex domain in section 5 , and theorem 2 is proved. In section 6 , we show that theorem 3 is true, using a Harnack type inequality. In section 7, we summarise what remains to be done on this problem.

For simplicity we denote the first $(N-1)$ coordinates by $\mathbf{x}^{\prime}$ and the last coordinate $x_{N}$ by $y$. Hence $\mathbf{x}=\left(x_{1}, x_{2}, \ldots, x_{N-1}, x_{N}\right)=\left(\mathbf{x}^{\prime}, y\right)$. Moreover let $b=a_{N}$.

Since $\Omega$ is bounded, we can make a translation of the domain so that it lies in the interior of the strip $\mathbf{R}^{N-1} \times[0, L]$ for some $L>0$. Let $0<m \leq p(\mathbf{x}) \leq M$. Define $\psi$ as the solution of the ordinary differential equation

$$
\begin{aligned}
& \psi_{y y}+\frac{M+1}{\psi^{b}}=0, \\
& \psi(0)=\psi(L)=0 .
\end{aligned}
$$


By the main theorem in [5] (which is restated in theorem 2 in [2]), a unique positive solution $\psi$ of (1.5)-(1.6) exists in $C^{2}(0, L) \cap C[0, L]$.

\section{An APPROXIMATION PROBLEM}

We want to study a regularized equation which approximates equation (1.3) in some sense. Let $r_{i}=a_{i}-a_{N}$ for $i=1,2, \ldots, N-1$. Therefore $r_{i} \geq 0$ and $r_{1}>0$. For any $\epsilon>0$, consider the equation

$$
\sum_{i=1}^{N-1}(u+\epsilon)^{r_{i}} \frac{\partial^{2} u}{\partial x_{i}^{2}}+u_{y y}+\frac{p(\mathbf{x})}{(u+\epsilon)^{b}}=0,
$$

subject to the boundary condition

$$
\left.u\right|_{\partial \Omega}=0
$$

But to study this quasilinear approximation problem, we have to first introduce a related semilinear equation. Define $S=\{v \in C(\bar{\Omega}): v=0$ on $\partial \Omega, 0 \leq v \leq$ $\psi$ on $\bar{\Omega}\}$. Hence $S$ is a closed bounded convex set in $C(\bar{\Omega})$. Given $v \in S$, we consider the semilinear equation

$$
\sum_{i=1}^{N-1}(v+\epsilon)^{r_{i}} \frac{\partial^{2} w}{\partial x_{i}^{2}}+w_{y y}+\frac{p(\mathbf{x})}{(w+\epsilon)^{b}}=0,
$$

subject to the boundary condition

$$
\left.w\right|_{\partial \Omega}=0 .
$$

Lemma 1. Let $\Omega$ be a bounded domain in $\mathbf{R}^{N}$ with smooth boundary $\partial \Omega$ (of class $C^{2+\alpha}$, where $\left.0<\alpha<1\right)$. If $p \in C^{\alpha}(\bar{\Omega}), M \geq p(\mathbf{x}) \geq m>0$ for all $\mathbf{x} \in \bar{\Omega}$, then for any $q>N$, there exists a unique nonnegative solution $w \in W^{2, q}(\Omega)$ for equations (2.3)-(2.4), and it satisfies $\psi(\mathbf{x}) \geq w(\mathbf{x}) \geq 0$ for all $\mathbf{x} \in \Omega$. Furthermore,

$$
\|w\|_{W^{2, q}} \leq C(\epsilon, \delta, q),
$$

where $C(\epsilon, \delta, q)$ is a positive constant which can depend on $\epsilon$, the modulus of continuity $\delta$ of $v$, and $q$.

Proof. The monotone iteration technique works in $W^{2, q}$ for our case. This is well known and is a slight modification of the proof in [8]. We include a brief outline here. The details are similar to the discussion leading to p.26 in [8].

Since $\varphi=0$ is a lower solution and $\psi$ as defined by equations (1.5)-(1.6) is an upper solution, monotone iteration [8] still works because the Aleksandrov maximum principle (theorem 9.1 in [4]) can be used to replace the classical maximum principle when $q \geq N$. Starting from the lower solution, we have a monotone increasing sequence $w_{n} \in W^{2, q}, q>1$, with a uniform upper bound. Here $w_{0}=\varphi=0$ and, for $n=0,1,2, \ldots$,

$$
\begin{aligned}
\sum_{i=1}^{N-1}(v+\epsilon)^{r_{i}} \frac{\partial^{2} w_{n+1}}{\partial x_{i}^{2}}+\frac{\partial^{2} w_{n+1}}{\partial y^{2}}-\lambda w_{n+1} & =-\lambda w_{n}-\frac{p(\mathbf{x})}{\left(w_{n}+\epsilon\right)^{b}}, \\
\left.w_{n+1}\right|_{\partial \Omega} & =0,
\end{aligned}
$$

where $\lambda=b M / \epsilon^{b+1}$. Without loss of generality we let $\epsilon \leq 1$.

Let $w_{n} \rightarrow w$ in a pointwise sense. From the $L^{q}$ theory of elliptic equations, this sequence will have a uniform bound in $W^{2, q}(\Omega)$ for any $N \leq q<\infty$. By taking $q>N$, the Sobolev theorem on compact embedding will ensure that a subsequence 
converges in the Hölder norm of $C^{\beta}(\bar{\Omega})$ for $\beta=1-N / q$, and hence converges uniformly. But it has a unique limit $w$. Thus the original sequence (not only a subsequence) must converge uniformly to $w$. Hence $\left\{w_{n}\right\}$ is a Cauchy sequence in the uniform norm. Elliptic regularity of equations (2.6) ensures that $\left\{w_{n}\right\}$ will be a Cauchy sequence in $W^{2, q}$ for any $q>N$. Thus it converges to $w$ in $W^{2, q}$. Since $q>N$, therefore $w \in C(\bar{\Omega})$. Thus there exists a $w \in W^{2, q}$ which satisfies equations (2.3) and (2.4). Moreover, $0 \leq w \leq \varphi$ by the construction. Furthermore, the Aleksandrov maximum principle will ensure that this equation has a unique non-negative solution.

By treating the nonlinear term in equation (2.3) as a given source term, the equation becomes linear. We then note that $w \in W^{2, q}(\Omega) \cap W_{0}^{1, q}(\Omega)$ (see theorem 9.15 on p. 241 of [4]). We can now employ lemma 9.17 on p. 242 of [4] to conclude that

$$
\|w\|_{W^{2, q}} \leq \frac{\tilde{C}(\epsilon, \delta, q)}{\epsilon^{b}}\|p\|_{\infty} \leq C(\epsilon, \delta, q)
$$

where $\tilde{C}(\epsilon, \delta, q)$ and $C(\epsilon, \delta, q)$ are positive constants which can depend on $\epsilon$, the modulus of continuity $\delta$ of $v$, and $q$. This completes the proof of lemma 1 .

We now return to study the equations (2.1)-(2.2).

Lemma 2. Let $\Omega$ be a bounded domain in $\mathbf{R}^{N}$ with smooth boundary $\partial \Omega$ (of class $C^{2+\alpha}$, where $0<\alpha<1$ ). If $p \in C^{\alpha}(\bar{\Omega}), M \geq p(\mathbf{x}) \geq m>0$ for all $\mathbf{x} \in \bar{\Omega}$, then there exists a solution $u_{\epsilon} \in C^{2+\alpha}(\bar{\Omega})$ for equations (2.1)-(2.2). Furthermore, $\psi(\mathbf{x}) \geq u_{\epsilon}(\mathbf{x}) \geq 0$ for all $\mathbf{x} \in \Omega$.

Proof. Define $T: S \rightarrow S$ such that for all $v \in S, T v=w$ as defined by equations (2.3)-(2.4). Since for any given $\epsilon>0$ the equation (2.3) is uniformly elliptic with

$$
\epsilon^{r_{1}} \leq \lambda_{\min } \leq \lambda_{\max } \leq 1+(M+\epsilon)^{r_{1}}
$$

and the nonlinear source term $\left\|p /(w+\epsilon)^{b}\right\|_{\infty} \leq M / \epsilon^{b}$, it follows that we can employ the global Hölder's estimate (corollary 9.29 on p. 252 of [4]) to conclude that

$$
\|T v\|_{C^{\beta}(\bar{\Omega})} \leq k_{\epsilon},
$$

where $0<\beta<1$ and $k_{\epsilon}$ are positive constants which can depend on $\epsilon$, but are uniform for all $v \in S$. The estimate implies that $T$ is a compact operator.

Deferring the proof of the continuity of $T$, we can employ the Schauder fixed point theorem in the closed bounded convex set $S$ to conclude that there is a fixed point $u_{\epsilon}$ of $T$. Treating the nonlinear term as an $L^{\infty}$ source term and employing $L^{q}$ elliptic regularity, we see that the solution $u_{\epsilon} \in W^{2, q}$ for any $1<q<\infty$. By taking $q$ large, we see that the solution $u_{\epsilon} \in C^{1+\alpha}(\bar{\Omega})$ for any $0<\alpha<1$. An application of Schauder's estimate gives $u_{\epsilon} \in C^{2+\alpha}(\bar{\Omega})$. This completes the proof of lemma 2 , provided we establish the continuity of $T$. This is done as follows.

For simplicity we rewrite the equation (2.3) as

$$
\sum_{i, j} a_{i j}(v) D_{i j} T v+g(\mathbf{x}, T v)=0
$$

where $g(\mathbf{x}, u)=p(\mathbf{x}) /(u+\epsilon)^{b}$. Let $v_{m} \in S$ and $v_{m} \rightarrow v$ in the uniform norm. Then

$$
\sum_{i, j} a_{i j}\left(v_{m}\right) D_{i j} T v_{m}+g\left(\mathbf{x}, T v_{m}\right)=0
$$


Note that $a_{i j}(v)$ and $a_{i j}\left(v_{m}\right)$ can be bounded by the same ellipticity constants in equation (2.7), which depend only on $\epsilon$ and $M$. On subtracting one equation from the other, we have, using mean value theorem on $g$,

$$
\sum_{i, j} a_{i j}\left(v_{m}\right) D_{i j}\left(T v-T v_{m}\right)-h(\mathbf{x})\left(T v-T v_{m}\right)=-\sum_{i, j}\left(a_{i j}(v)-a_{i j}\left(v_{m}\right)\right) D_{i j} T v,
$$

where $h \geq 0$ for all $\mathbf{x}$ and is bounded. Since $T v-T v_{m}$ satisfies the zero Dirichlet boundary condition and is in $W^{2, q}$ with $q>N$, we can apply the Aleksandrov maximum principle (theorem 9.1 on p. 220 of [4]) to obtain

$$
\begin{aligned}
\left\|T v-T v_{m}\right\|_{\infty} & \leq C_{\epsilon} \sum_{i, j}\left\|\left(a_{i j}(v)-a_{i j}\left(v_{m}\right)\right) D_{i j} T v\right\|_{L^{N}} \\
& \leq C_{\epsilon}\left\|a_{i j}(v)-a_{i j}\left(v_{m}\right)\right\|_{\infty}\|T v\|_{W^{2, N}} \rightarrow 0 \text { as } m \rightarrow \infty
\end{aligned}
$$

because of the estimate (2.5). Here $C_{\epsilon}$ is a constant which depends on $\epsilon$ through the elliptic constants for $a_{i j}\left(v_{m}\right)$. Hence $T v_{m}$ converges to $T v$ uniformly. Thus $T$ is continuous.

\section{A COMPARISON LEMMA}

In this section, using the maximum principle we will establish a comparison lemma on a class of quasilinear elliptic equations. This allows us to obtain $L^{\infty}$ bounds on the solutions to this class of equations, using the method of upper and lower solutions. The proof is similar to that of lemma 1 in [2], and hence will be skipped.

Lemma 3. Given the equation

$$
\sum_{i=1}^{N-1} f_{i}(u, \mathbf{x}) \frac{\partial^{2} u}{\partial x_{i}^{2}}+u_{y y}+p(\mathbf{x}) g(u, \mathbf{x})=0,
$$

subject to the boundary condition

$$
\left.u\right|_{\partial \Omega}=\left.u_{0}\right|_{\partial \Omega},
$$

with $\left.u_{0}\right|_{\partial \Omega} \geq 0$, let $f_{i}, g$, and $p$ satisfy the conditions (A1)-(A3) respectively, where

(A1) $f_{i}:[0, \infty) \times \Omega \rightarrow[0, \infty)$ is continuous, and $f(\cdot, \mathbf{x})$ is non-decreasing for each $\mathbf{x} \in \Omega$.

(A2) $g:(0, \infty) \times \Omega \rightarrow(0, \infty)$ is continuous, and $g(\cdot, \mathbf{x})$ is non-increasing for each $\mathbf{x} \in \Omega$.

(A3) $p: \bar{\Omega} \rightarrow \mathbf{R}$ is continuous, and there exist positive constants $m$ and $M$ such that $0<m \leq p(\mathbf{x}) \leq M$ for all $\mathbf{x} \in \bar{\Omega}$.

Further, we make the following assumptions:

(L) There exists a lower solution $\varphi \in C(\bar{\Omega}) \cup C^{2}(\Omega)$ with $\varphi>0$ on $\Omega$ satisfying

$$
\begin{gathered}
\sum_{i=1}^{N-1} f_{i}(\varphi, \mathbf{x}) \frac{\partial^{2} \varphi}{\partial x_{i}^{2}}+\varphi_{y y}+p(\mathbf{x}) g(\varphi, \mathbf{x})>0, \\
\frac{\partial^{2} \varphi}{\partial x_{i}^{2}} \leq 0, \quad i=1,2, \ldots, N-1,
\end{gathered}
$$

on $\Omega$, and $\left.\varphi\right|_{\partial \Omega} \leq\left. u_{0}\right|_{\partial \Omega}$. [If $\varphi \geq \delta>0$ on $\bar{\Omega}$ for some $\delta>0$, we can allow $\geq$ in (3.3).] 
(U) There exists an upper solution $\psi \in C(\bar{\Omega}) \cup C^{2}(\Omega)$ with $\psi>0$ on $\Omega$ satisfying

$$
\begin{gathered}
\sum_{i=1}^{N-1} f_{i}(\psi, \mathbf{x}) \frac{\partial^{2} \psi}{\partial x_{i}^{2}}+\psi_{y y}+p(\mathbf{x}) g(\psi, \mathbf{x}) \leq 0 \\
\frac{\partial^{2} \psi}{\partial x_{i}^{2}} \leq 0, \quad i=1,2, \ldots, N-1
\end{gathered}
$$

on $\Omega$, and $\left.\psi\right|_{\partial \Omega} \geq\left. u_{0}\right|_{\partial \Omega}$.

If $u \in C(\bar{\Omega}) \cup C^{2}(\Omega)$ is any solution of equations (1.5) and (1.6) with $u>0$ on $\Omega$, then

$$
\varphi \leq u \leq \psi
$$

on $\bar{\Omega}$.

All remarks 1 to 3 in [2] hold. In particular we have the following.

Remark 1. If only conditions (A1)-(A3) and (L) hold, the proof for the lemma still gives $\varphi \leq u$ on $\bar{\Omega}$. The similar remark applies to the upper solution $\psi$.

\section{Proof of Theorem 1}

In order to extract a solution to equations (1.3)-(1.4) from the sequence $\left\{u_{\epsilon}\right\}$, we need to show that the sequence is bounded away from zero in any compact subset of $\Omega$. We prove this fact by our comparison lemma. Without loss of generality, we let $\epsilon \leq 1$ and $R \leq 1$ in the following.

For any $\mathbf{x}_{0} \in \Omega$, we take an open ball $S$ centered at $\mathbf{x}_{0}$ with $S \subset \subset \Omega$. Without loss of generality we can regard $\mathbf{x}_{0}$ as the origin. Letting the radius of $S$ be $R$ and $\delta>0$, we then define

$$
\varphi=\delta\left(R^{2}-\sum_{i=1}^{N} x_{i}^{2}\right) .
$$

Hence $\left.\varphi\right|_{\partial S}=0, \varphi>0$ on $S$, and $\partial^{2} \varphi / \partial x_{i}^{2} \leq 0$ for $i=1, \ldots, N$. Moreover for $\mathbf{x} \in S$,

$$
\begin{aligned}
& \sum_{i=1}^{N-1}(\varphi+\epsilon)^{r_{i}} \frac{\partial^{2} \varphi}{\partial x_{i}^{2}}+\varphi_{y y}+\frac{p(\mathbf{x})}{(\varphi+\epsilon)^{b}} \\
& \geq-2 \delta \sum_{i=1}^{N-1}\left(\delta R^{2}+\epsilon\right)^{r_{i}}-2 \delta+\frac{m}{\left(\delta R^{2}+\epsilon\right)^{b}} \\
& \quad \geq-2 \delta \sum_{i=1}^{N-1}(\delta+1)^{r_{i}}-2 \delta+\frac{m}{(\delta+1)^{b}} \\
& \quad>0
\end{aligned}
$$

by choosing $\delta=\delta\left(m, r_{i}, b\right)=\delta\left(m, a_{1}, \ldots, a_{N}\right)$ sufficiently small. Hence $\varphi$ is a strict lower solution for equation (2.1). Thus, employing lemma 3 on the ball $S$, we conclude that

$$
\delta\left(R^{2}-\sum_{i=1}^{N} x_{i}^{2}\right) \leq u_{\epsilon} \leq \psi
$$

on any ball $\bar{S}$ of radius $R$ for any $\epsilon$, provided $S \subset \Omega$. 
We now restrict our attention to the domain $3 S / 4$, which is the ball centered at the origin with radius $3 R / 4$. We treat equations (2.1)-(2.2) as a linear equation. From equation (4.2), inside the ball $3 S / 4$ the equation is uniformly elliptic with

$$
\left(\frac{7}{16} \delta R^{2}\right)^{r_{1}} \leq \lambda_{\min } \leq \lambda_{\max } \leq 1+(M+1)^{r_{1}}
$$

We can now apply the interior Hölder estimate to equation (2.1) in the ball $S / 2$ (corollary 9.24 on p. 250 of [4]) to get

$$
\left\|u_{\epsilon}\right\|_{C^{\gamma}(\overline{S / 2})} \leq C_{1}
$$

for some positive constants $0<\gamma<1$ and $C_{1}$, which can depend on $R$ but are independent of $\epsilon$. Further bootstrapping using the interior Schauder estimate (theorem 6.2 on p. 90 of [4]) in the ball $S / 4$, we can obtain

$$
\left\|u_{\epsilon}\right\|_{C^{2+\gamma}(\overline{S / 4})} \leq C_{2}\left(\left\|u_{\epsilon}\right\|_{L^{\infty}(\overline{S / 2})}+\left\|p / u_{\epsilon}^{b}\right\|_{C^{\gamma}(\overline{S / 2})}\right) \leq k_{1},
$$

where $C_{2}$ can depend on the $C^{\gamma}(\overline{S / 2})$ bound on $u_{\epsilon}$, and $k_{1}=k_{1}\left(M, m, R, a_{1}, \ldots, a_{N}\right)$ for any $\epsilon$. Hence there exists a subsequence $u_{\epsilon} \rightarrow v$ in $C^{2}(\overline{S / 4})$. It is also clear from equation (4.2) that $v(\mathbf{x}) \geq \frac{15}{16} \delta R^{2}>0$ for $\mathbf{x} \in \overline{S / 4}$. Since $u_{\epsilon}$ is a solution of (2.1), $v$ will satisfy

$$
\sum_{i=1}^{N-1} v^{r_{i}} \frac{\partial^{2} v}{\partial x_{i}^{2}}+v_{y y}+\frac{p(\mathbf{x})}{v^{b}}=0,
$$

on $\overline{S / 4}$. Now we can cover any compact subset $\Omega_{1}$ of $\Omega$ with finite number of balls $S_{i}$ such that $4 S_{i}$ still lies inside $\Omega$. The previous argument immediately gives a subsequence of $u_{\epsilon}$ such that they converge to $v \in C^{2}\left(\overline{\Omega_{1}}\right)$ with $v>0$ satisfying equation (4.6). Let $\Omega_{1} \rightarrow \Omega$. By taking a diagonal subsequence if necessary, $v \in$ $C^{2}(\Omega)$ will satisfy the equation (4.6) on the whole domain $\Omega$. Moreover, $\psi \geq v>0$ on $\Omega$. A standard regularity estimate on equation (4.6) gives $v \in C^{2+\alpha}(\Omega)$.

Remark 2. From the construction up to now in this section, we see that there exists a solution $v$ of equation (1.3) in $C^{2+\alpha}(\Omega)$ such that a sequence $u_{\epsilon}$ converges to $v$ in $C^{2}(\Omega)$ and $0<v \leq \psi$ on $\Omega$.

In order for $v$ to qualify as a classical solution of equations (1.3)-(1.4), we have to show that $v \in C(\bar{\Omega})$ and $\left.v\right|_{\partial \Omega}=0$. This is the case for a convex domain.

Let $\Omega$ be convex in the rest of this section. Then for any $\mathbf{x} \in \partial \Omega$, there is a tangent plane $T_{\mathbf{x}}$ passing through $\mathbf{x}$ with $\Omega$ lying on one side of $T_{\mathbf{x}}$. We can also construct another plane $T_{\mathbf{x}}^{\prime}$ parallel to $T_{\mathbf{x}}$ such that $\Omega$ is contained in the semiinfinite strip enclosed by $T_{\mathbf{x}}$ and $T_{\mathbf{x}}^{\prime}$. By translation we can let the origin lies in $T_{\mathbf{x}}$. Take coordinate axes $X_{1}, \ldots, X_{N-1}$ and $y_{1}$ so that the $y_{1}$ axis is perpendicular to $T_{\mathbf{x}}$. Let $\beta_{i}$ be the angle that the $y_{1}$ axis makes with the $x_{i}$ axis for $i=1, \ldots, N-1$, and $\beta$ be the angle that the $y_{1}$ axis makes with the $y$ axis. It can be checked that $y_{1}=\sum_{i=1}^{N-1} x_{i} \cos \beta_{i}+y \cos \beta$.

We will distinguish two cases.

Case (i): The $y$ axis and the $y_{1}$ axis are not perpendicular to one another. Thus $\beta \neq \pi / 2$ or $3 \pi / 2$. Throughout case (i), $\beta$ will be fixed.

Define $\bar{\phi}=\bar{\phi}\left(y_{1}\right)$ such that it solves

$$
\frac{\partial^{2} \bar{\phi}}{\partial y_{1}^{2}}+\frac{M}{\bar{\phi}^{b}}=0,
$$


subject to the boundary conditions

$$
\left.\bar{\phi}\right|_{T_{\mathbf{x}}}=0,\left.\quad \bar{\phi}\right|_{T_{\mathbf{x}}^{\prime}}=0 .
$$

Hence $\bar{\phi} \in C(\bar{\Omega}) \cap C^{2}(\Omega), \bar{\phi}>0$, and $\partial^{2} \bar{\phi} / \partial y_{1}^{2} \leq 0$ on $\Omega$.

Let $\phi(x, y)=\bar{\phi}\left(y_{1}\left(\mathbf{x}^{\prime}, y\right)\right) / \cos ^{2} \beta$. Simple calculations show that for $i=1, \ldots, N-$ $1, \partial^{2} \phi / \partial x_{i}^{2}=\frac{\cos ^{2} \beta_{i}}{\cos ^{2} \beta} \frac{\partial^{2} \bar{\phi}}{\partial y_{1}^{2}} \leq 0$, and $\partial^{2} \phi / \partial y^{2}=\partial^{2} \bar{\phi} / \partial y_{1}^{2}$. By lemma 3 the function $\phi$ is an upper solution for equation (2.1) for any $\epsilon>0$, because

$$
\begin{aligned}
\sum_{i=1}^{N-1} & (\phi+\epsilon)^{r_{i}} \frac{\partial^{2} \phi}{\partial x_{i}^{2}}+\phi_{y y}+p(\mathbf{x}) /(\phi+\epsilon)^{b} \\
& \leq \phi_{y y}+p(\mathbf{x}) / \phi^{b} \\
& =\partial^{2} \bar{\phi} / \partial y_{1}^{2}+p(\mathbf{x})(\cos \beta)^{2 b} / \bar{\phi}^{b} \\
& \leq-M / \bar{\phi}^{b}+p(\mathbf{x}) / \bar{\phi}^{b} \\
& \leq 0 .
\end{aligned}
$$

Hence by lemma 3 (and remark 1 in section 3 ), we have $u_{\epsilon} \leq \phi$ on $\bar{\Omega}$. By remark 2 , we can take the limit as $\epsilon \rightarrow 0$ to obtain

$$
0<v \leq \phi \text { on } \Omega \text {. }
$$

Case (ii): The $y$ axis and the $y_{1}$ axis are perpendicular to one another.

Define $\phi=\phi(\mathbf{x})$ such that it solves

$$
\phi^{r_{1}} \frac{\partial^{2} \phi}{\partial x_{1}^{2}}+\frac{M}{\phi^{b}}=0
$$

subject to the boundary conditions

$$
\left.\phi\right|_{T_{\mathbf{x}}}=0,\left.\quad \phi\right|_{T_{\mathbf{x}}^{\prime}}=0 .
$$

Hence $\sum_{i=1}^{N-1}(\phi+\epsilon)^{r_{i}} \frac{\partial^{2} \phi}{\partial x_{i}^{2}}+\phi_{y y}+p(\mathbf{x}) /(\phi+\epsilon)^{b} \leq 0$, and equation (4.9) holds.

Now in both cases, define $\left.v\right|_{\partial \Omega}=0$. Take any $\mathbf{x} \in \partial \Omega$ and $\mathbf{y} \in \Omega$. Since

$$
|v(\mathbf{y})-v(\mathbf{x})|=v(\mathbf{y}) \leq \phi(\mathbf{y}) \rightarrow 0
$$

as $\mathbf{y} \rightarrow \mathbf{x}$, hence $v$ is continuous at $\mathbf{x} \in \partial \Omega$. Since $\mathbf{x}$ can be any arbitrary point on $\partial \Omega, v \in C(\bar{\Omega}) \cap C^{2}(\Omega)$, and it satisfies both the equation (1.3) and the boundary condition (1.4). This completes the proof of theorem 1.

\section{WEAK SOLUTION IN NON-CONVEX DOMAINS}

For non-convex domains, we cannot construct an upper solution $\phi$ as in the last section. Hence an energy estimate is performed to establish the existence of a weak solution. This result has been stated in theorem 2 .

Remark 3. It may be tricky to construct an upper solution which forces our constructed solution to attain a value of zero at the boundary. Consider the approximation problem (see equations (2.1)-(2.2))

$$
(u+\epsilon) u_{x x}+u_{y y}+1=0
$$

in the annulus domain $\left\{(x, y) \in \mathbf{R}^{2}: 1<x^{2}+y^{2}<4\right\}$. If the upper solution $\phi$ is smooth up to the boundary, then at the point $(0,1)$ we require $\phi_{x x} \leq 0$ according to condition (U) in the comparison lemma. However, since $\phi=0$ at this point, it 
attains its minimum in the $x$-direction there, and hence $\phi_{x x} \geq 0$ at $(0,1)$. Thus $\phi_{x x}=0$ at $(0,1)$. These impose stringent requirements on $\phi$.

Proof of theorem 2. From lemma 2, we have a sequence of positive solutions $\left\{u_{\epsilon}\right\} \in$ $C^{2+\alpha}(\bar{\Omega})$ which satisfy

$$
\begin{gathered}
\sum_{i=1}^{N}\left(u_{\epsilon}+\epsilon\right)^{a_{i}} \frac{\partial^{2} u_{\epsilon}}{\partial x_{i}^{2}}+p(\mathbf{x})=0, \\
\left.u_{\epsilon}\right|_{\partial \Omega}=0 .
\end{gathered}
$$

Moreover, $0 \leq u_{\epsilon} \leq \psi$ on $\bar{\Omega}$. Let $K=\|\psi\|_{\infty}$, and define $\gamma_{i}=\left(1+a_{i}\right) / 2$ for $i=1, \ldots, N$. Then $u_{\epsilon}^{\gamma_{1}} \in H_{0}^{1}(\Omega)$, and the sequence $\left\{u_{\epsilon}\right\}$ has a uniform $L^{\infty}(\Omega)$ bound. Let $\mathbf{n}(\mathbf{x})$ be the unit outward normal at $\mathbf{x} \in \partial \Omega$, and $\sum_{+}=\sum_{i=1, a_{i}>0}^{N}$, to simplify our notation.

We perform an integration by parts on equations (5.1)-(5.2) to obtain

$$
\sum_{i=1}^{N} \int_{\partial \Omega}\left(u_{\epsilon}+\epsilon\right)^{a_{i}} \frac{\partial u_{\epsilon}}{\partial x_{i}} n_{i}-\sum_{+} \int_{\Omega} a_{i}\left(u_{\epsilon}+\epsilon\right)^{a_{i}-1}\left(\frac{\partial u_{\epsilon}}{\partial x_{i}}\right)^{2}+\int_{\Omega} p=0 .
$$

Since at any $\mathbf{x} \in \partial \Omega, u_{\epsilon}$ attains its minimum value 0 , it follows that $\frac{\partial u_{\epsilon}}{\partial x_{i}} n_{i} \leq 0$ for each $i=1, \ldots, N$. We therefore have

$$
\sum_{+} a_{i} \int_{\Omega}\left(u_{\epsilon}+\epsilon\right)^{a_{i}-1}\left(\frac{\partial u_{\epsilon}}{\partial x_{i}}\right)^{2} \leq \int_{\Omega} p
$$

This gives

$$
\sum_{+} \frac{a_{i}}{\gamma_{i}^{2}} \int_{\Omega}\left(\frac{\partial u_{\epsilon}^{\gamma_{i}}}{\partial x_{i}}\right)^{2}=\sum_{+} a_{i} \int_{\Omega}\left(u_{\epsilon}\right)^{a_{i}-1}\left(\frac{\partial u_{\epsilon}}{\partial x_{i}}\right)^{2} \leq M|\Omega| .
$$

We now claim that $u_{\epsilon}^{\gamma_{1}}$ has a uniform bound in $H_{0}^{1}$.

Case (1): For any $i$ such that $a_{i}>0$, since $\gamma_{i} \leq \gamma_{1}$,

$$
\begin{aligned}
\int_{\Omega}\left(\frac{\partial u_{\epsilon}^{\gamma_{1}}}{\partial x_{i}}\right)^{2} & =\gamma_{1}^{2} \int_{\Omega}\left(u_{\epsilon}\right)^{a_{1}-1}\left(\frac{\partial u_{\epsilon}}{\partial x_{i}}\right)^{2} \\
& \leq \gamma_{1}^{2} K^{a_{1}-a_{i}} \int_{\Omega}\left(u_{\epsilon}\right)^{a_{i}-1}\left(\frac{\partial u_{\epsilon}}{\partial x_{i}}\right)^{2} \\
& \leq \gamma_{1}^{2} K^{a_{1}-a_{i}} M|\Omega| / a_{i},
\end{aligned}
$$

where the last inequality follows from equation (5.3).

Case (2): For any $i$ such that $a_{i}=0$, observe that $\int_{\Omega}\left(u_{\epsilon}+\epsilon\right)^{a_{1}} p$ is bounded uniformly in $\epsilon$. Thus we can multiply equation (5.1) by $\left(u_{\epsilon}+\epsilon\right)^{a_{1}}$ and integrate. Arguing as in case (1) above, we obtain equation (5.4) for $i$ in this case.

Combining cases (1) and (2), we establish a uniform bound on the $H_{0}^{1}(\Omega)$ bound on $\left\{u_{\epsilon}^{\gamma_{1}}\right\}$.

By the $H^{1}$ norm uniform bound that we just established, there exists a $w \in$ $H_{0}^{1}(\Omega)$ such that a subsequence $u_{\epsilon}^{\gamma_{1}} \rightarrow w$ weakly in $H_{0}^{1}(\Omega)$. Hence $u_{\epsilon}^{\gamma_{1}} \rightarrow w$ pointwise a.e. But by remark 2, we know that $u_{\epsilon}^{\gamma_{1}} \rightarrow v^{\gamma_{1}}$ pointwise, where $\psi \geq v>0$ is a solution to equation (1.3). Thus $w=v^{\gamma_{1}}$. The proof of theorem 2 is now complete. 


\section{Boundary CONTINUITY OF SOLUTIONS}

In the last section a weak solution was constructed for equations (1.3)-(1.4) in smooth bounded non-convex domains. This solution is smooth in the interior and satisfies the boundary conditions only in a weak sense. In some special cases we are able to show that it is continuous up to the boundary and hence is a classical solution.

Let $N=2$ and $a \equiv a_{1}+a_{2}<1$. From lemma 2 there is a solution $u_{\epsilon} \in C^{2+\alpha}(\bar{\Omega})$ that satisfies

$$
\begin{gathered}
\left(u_{\epsilon}+\epsilon\right)^{a_{1}} \frac{\partial^{2} u_{\epsilon}}{\partial x_{1}^{2}}+\left(u_{\epsilon}+\epsilon\right)^{a_{2}} \frac{\partial^{2} u_{\epsilon}}{\partial x_{2}^{2}}+p(\mathbf{x})=0 \\
\left.u_{\epsilon}\right|_{\partial \Omega}=0
\end{gathered}
$$

Recall from remark 2 that $u_{\epsilon} \rightarrow v$ in $C^{2}(\Omega)$ and $v$ satisfies equation (1.3). We would like to show that $v(x) \rightarrow 0$ as $x \rightarrow \partial \Omega$.

We will use a Harnack type inequality to show this fact. It is essentially the same calculations as in the proof of theorems 9.20 and 9.26 in [4]. The only difference is that we are dealing with the case that the equation is not uniformly elliptic and have to bookkeep the important coefficients accordingly. For simplicity we write equation (6.1) as $L u_{\epsilon}+p=0$ and $L u=\sum a_{i j} D_{i j} u$. Thus $a_{11}=\left(u_{\epsilon}+\epsilon\right)^{a_{1}}$, $a_{22}=\left(u_{\epsilon}+\epsilon\right)^{a_{2}}$, and $a_{12}=a_{21}=0$. Let $K=\|\psi\|_{\infty}$. Hence $K$ is a uniform $L^{\infty}$ apriori bound on $u_{\epsilon}$, and there exists a positive constant $\Lambda=\Lambda\left(K, a_{1}, a_{2}\right)$, independent of $\epsilon$, such that $a_{i i} \leq \Lambda$ for $i=1,2$.

Lemma 4. For any ball $B_{2 R}(\mathbf{y}) \subset \Omega \subset \mathbf{R}^{2}$, we have

$$
\sup _{B_{R}(\mathbf{y})} u_{\epsilon} \leq C\left\{\left(\frac{1}{\left|B_{2 R}\right|} \int_{B_{2 R}} u_{\epsilon}\right)^{1 / 2}+R\left(\int_{B_{2 R}} \frac{1}{\left(u_{\epsilon}+\epsilon\right)^{a}}\right)^{1 / 2}\right\}
$$

where $C$ is a positive constant which depends on $\Lambda, M$ and $K$, but is independent of $R$ and $\epsilon$.

Proof. We follow the procedures for theorem 9.20 on p. 244 of [4]. Without loss of generality, we can assume that $2 R=1$ and $y$ is the origin. The general case will be recovered by the transformation $\mathbf{x} \rightarrow(\mathbf{x}-\mathbf{y}) / 2 R$.

On the ball $B_{1}$, we define $\eta=\left(1-|\mathbf{x}|^{2}\right)^{2}$ and $v=\eta u_{\epsilon}$. Direct calculations give

$$
\begin{aligned}
\sum_{i, j} a_{i j} D_{i j} v & =\sum_{i, j}\left[\eta a_{i j} D_{i j} u_{\epsilon}+2 a_{i j} D_{i} \eta D_{j} u_{\epsilon}+u_{\epsilon} a_{i j} D_{i j} \eta\right] \\
& =-\eta p+\sum_{i, j}\left[2 a_{i j} D_{i} \eta D_{j} u_{\epsilon}+u_{\epsilon} a_{i j} D_{i j} \eta\right] .
\end{aligned}
$$

From the calculations in [4], on the upper contact set $\Gamma_{v}^{+}$of $v$ in the ball $B_{1}$ we have

$$
\left|\nabla u_{\epsilon}\right| \leq 4 \eta^{-1 / 2} u_{\epsilon}
$$

Furthermore it can be checked that

$$
\begin{aligned}
D_{i} \eta & =-4 x_{i}\left(1-|\mathbf{x}|^{2}\right) \\
D_{i j} \eta & =-4 \delta_{i j}\left(1-|\mathbf{x}|^{2}\right)+8 x_{i} x_{j} .
\end{aligned}
$$


Hence on the contact set $\Gamma_{v}^{+}$, equation (6.4) becomes

$$
\begin{aligned}
-\sum_{i, j} a_{i j} D_{i j} v & \leq p+2 \Lambda|\nabla \eta|\left|\nabla u_{\epsilon}\right|+4 u_{\epsilon}\left(1-|\mathbf{x}|^{2}\right)\left(a_{11}+a_{22}\right) \\
& \leq p+32 \Lambda u_{\epsilon}+8 \Lambda u_{\epsilon} \eta^{1 / 2} \\
& \leq p+40 \Lambda u_{\epsilon} .
\end{aligned}
$$

By lemma 9.3 on p. 222 of [4] on $v$ with diameter $\left(B_{1}\right)=2$ and $v=0$ on $\partial B_{1}$, there exists a constant $C=1 / \sqrt{\pi}$ such that

$$
\sup _{B_{1}} v \leq C\left\|\frac{\sum_{i, j} a_{i j} D_{i j} v}{\mathcal{D}}\right\|_{L^{2}\left(\Gamma_{v}^{+}\right)},
$$

where $\mathcal{D}=\left(u_{\epsilon}+\epsilon\right)^{a / 2}$. In what follows we use $C$ to denote any generic constant which may depend on $\Lambda, M$ and $K$. Thus, combining the previous two equations,

$$
\begin{aligned}
\sup _{B_{1}} v \leq C\left[\left\|\frac{p}{\mathcal{D}}\right\|_{L^{2}\left(B_{1}\right)}+\left\|\frac{u_{\epsilon}}{\mathcal{D}}\right\|_{L^{2}\left(B_{1}\right)}\right] \\
\leq C\left[\left(\int_{B_{1}}\left(u_{\epsilon}+\epsilon\right)^{-a}\right)^{1 / 2}+\left(\int_{B_{1}} u_{\epsilon}^{2-a}\right)^{1 / 2}\right] \\
\leq C\left[\left(\int_{B_{1}}\left(u_{\epsilon}+\epsilon\right)^{-a}\right)^{1 / 2}+\left(\int_{B_{1}} u_{\epsilon}\right)^{1 / 2}\right],
\end{aligned}
$$

where the last inequality follows because of the uniform $L^{\infty}$ bound on $u_{\epsilon}$. With

$$
\sup _{B_{1 / 2}} u_{\epsilon} \leq\left(\frac{16}{15}\right)^{2} \sup _{B_{1}} v
$$

the proof of lemma 4 is now complete.

With balls centered at $\partial \Omega$, one can argue as in the proof of theorem 9.26 on p. 250 of [4] by extending $u_{\epsilon}$ to be zero outside $\Omega$, and obtain the following key boundary estimate:

Lemma 5. Let $\Omega \subset \mathbf{R}^{2}$ be a bounded domain with smooth boundary $\partial \Omega$ (of class $\left.C^{2+\alpha}, 0<\alpha<1\right)$, and let $y \in \partial \Omega$. Then for any ball $B_{2 R}(\mathbf{y})$, we have

$$
\sup _{\Omega \cap B_{R}(\mathbf{y})} u_{\epsilon} \leq C\left\{\left(\frac{1}{\left|B_{2 R}\right|} \int_{B_{2 R} \cap \Omega} u_{\epsilon}\right)^{1 / 2}+R\left(\int_{B_{2 R} \cap \Omega} \frac{1}{\left(u_{\epsilon}+\epsilon\right)^{a}}\right)^{1 / 2}\right\},
$$

where $C$ is a positive constant which depends on $\Lambda, M$ and $K$, but is independent of $R$ and $\epsilon$.

Take a ball of radius $R$. Since the boundary is $C^{2}$, there exists an $R_{0}$ such that if $R \leq R_{0}$ then for any $\mathbf{y} \in \partial \Omega$, the ball lies inside $\bar{\Omega}$ and touches the boundary of $\Omega$ only at the point $\mathbf{y}$. Hence from equation (4.2), we know that for all $\mathbf{x}$ such that $\operatorname{dist}(\mathbf{x}, \partial \Omega) \leq R_{0}$, there exists a $\delta_{1}>0$ such that

$$
u_{\epsilon}(\mathbf{x}) \geq \delta_{1} \operatorname{dist}(\mathbf{x}, \partial \Omega) \text {. }
$$

Hence, from the estimate (6.8),

$$
\sup _{\Omega \cap B_{R}(\mathbf{y})} u_{\epsilon} \leq C\left\{\left(\frac{1}{\left|B_{2 R}\right|} \int_{B_{2 R} \cap \Omega} u_{\epsilon}\right)^{1 / 2}+R\left(\int_{B_{2 R} \cap \Omega} \frac{1}{(\operatorname{dist}(\mathbf{x}, \partial \Omega))^{a}}\right)^{1 / 2}\right\} .
$$


We now pass the limit as $\epsilon \rightarrow 0$. Since $u_{\epsilon}$ converges in $C^{2}(\Omega)$ to $v$ and $u_{\epsilon}^{\theta}$ converges weakly in $H_{0}^{1}(\Omega)$ to $v^{\theta}$ for $\theta=\left(1+a_{1}\right) / 2$ (see the end of section 5 ), hence $u_{\epsilon}^{\theta}$ converges in $L^{2}(\Omega)$ to $v^{\theta}$. So there exists a subsequence, still labelled $\left\{u_{\epsilon}\right\}$, such that $u_{\epsilon}^{\theta}$ converges pointwisely a.e. to $v^{\theta}$. In other words, $u_{\epsilon}$ converges pointwisely a.e. to $v$. Because of the uniform $L^{\infty}$ norm bound, $u_{\epsilon} \rightarrow v$ in $L^{1}(\Omega)$ by the dominated convergence theorem. Therefore

$$
\sup _{\Omega \cap B_{R}(\mathbf{y})} v \leq C\left\{\left(\frac{1}{\left|B_{2 R}\right|} \int_{B_{2 R} \cap \Omega} v\right)^{1 / 2}+R\left(\int_{B_{2 R} \cap \Omega} \frac{1}{(\operatorname{dist}(\mathbf{x}, \partial \Omega))^{a}}\right)^{1 / 2}\right\} .
$$

Since $v^{\theta} \in H_{0}^{1}(\Omega)$ with $\theta<1$ and $v$ has an $L^{\infty}$ norm bound, a simple calculation shows that $v \in H_{0}^{1}(\Omega)$. After extending $v \in H_{0}^{1}(\Omega)$ to be zero outside $\Omega, v \in$ $H^{1}\left(B_{2 R}\right)$. Hence by Poincaré's lemma there exists a $k \geq 0$, independent of $R$, such that

$$
\begin{aligned}
\frac{1}{\left|B_{2 R}\right|} \int_{B_{2 R} \cap \Omega} v & \leq k\left(\frac{R^{2}}{\left|B_{2 R}\right|} \int_{B_{2 R}} \cap \Omega|\nabla v|^{2}\right)^{1 / 2} \\
& \leq k\left(\int_{B_{2 R} \cap \Omega}|\nabla v|^{2}\right)^{1 / 2} \rightarrow 0 \text { as } R \rightarrow 0,
\end{aligned}
$$

where the last assertion follows from the dominated convergence theorem.

For $a<1$, there exists a constant $k_{1}>0$ such that

$$
\int_{B_{2 R} \cap \Omega} \frac{1}{\left(\operatorname{dist}(\mathbf{x}, \partial \Omega)^{a}\right.}<k_{1} \text {. }
$$

Hence by equations (6.12) and (6.13), the right hand side of estimate (6.11) goes to zero as $R \rightarrow 0$. In other words,

$$
\sup _{\Omega \cap B_{R}(\mathbf{y})} v \rightarrow 0 \text { as } R \rightarrow 0 .
$$

Thus $v$ is continuous up to the boundary. The proof of theorem 3 is now complete.

\section{WHAT'S LEFT}

The most significant omission of this paper is the absence of any results on uniqueness. Although this is not a problem in the isotropic case, there is almost nothing known in the nonisotropic case. The only result, for a very restricted class of domains and a restricted class of functions $p$, occurs in [7].

The second outstanding problem is to reconcile the various existence results with the different hypotheses. It is natural to conjecture that the results of theorem 3 are true without restriction on the geometry of the domain, the size of the "degeneracy coeffients" $a_{i}$, or the dimension of the space.

Finally, one should expect results which allow for more general degenerate quasilinear operators and a less restricted class of functions $p$.

\section{REFERENCES}

1. S. C̆anić and B. L. Keyfitz, An elliptic problem arising form the unsteady transonic small disturbance equation, J. Diff. Eqs. 125 (1996), 548-574. MR 97c:35073

2. Y. S. Choi, A.C. Lazer, and P. J. McKenna, On a singular quasilinear anisotropic elliptic boundary value problem, Trans. A.M.S., 347 (1995), pp. 2633-2641. MR 95i:35087

3. M.G.Crandall, P. H. Rabinowitz, and L. Tartar, On a Dirichlet problem with a singular nonlinearity, Comm. P.D.E.,2(2), (1977) 193-222. MR 55:856 
4. D. Gilbarg and N. S. Trudinger. Elliptic Partial Differential Equations of Second Order. 2nd edition, (1983) Springer-Verlag. MR 86c:35035

5. A. C. Lazer and P. J. McKenna, On a singular nonlinear elliptic boundary value problem, Proceedings of the AMS, 111, (1991) 721-730. MR 91f:35099

6. A. Nachman and A. Callegari, A nonlinear singular boundary value problem in the theory of pseudoplastic fluids, SIAM J. Appl. Math. 28 (1986), 271-281. MR 81e:76003

7. W. Reichel, Uniqueness for degenerate elliptic equations via Serrin's sweeping principle, General Inequalities 7, International Series of Numerical Mathematics, Birkhäuser, Basel, 1997, pp. 375-387. CMP 97:14

8. D.H. Sattinger, Topics in Stability and Bifurcation Theory, Lecture Notes in Mathematics, no. 309, Springer-Verlag, 1973. MR 57:3569

9. C.A. Stuart, Existence theorems for a class of nonlinear integral equations, Math. Z., 137 (1974), 49-66. MR 50:914

10. S.D. Taliaferro, A nonlinear singular boundary value problem, Nonlinear Analysis, T.M.A. 3 (1979), 897-904. MR 81i:34011

Department of Mathematics, University of Connecticut, Storrs, Connecticut 06268

E-mail address: choi@math.uconn.edu

E-mail address: mckenna@math.uconn.edu 